\title{
The Status of Normative Propositions in the Theory of Scientific Change
}

\author{
Zoe Sebastien \\ IHPST, University of Toronto \\ zoe.sebastien@mail.utoronto.ca
}

An earlier version of this paper was awarded the 2015 IHPST Award for the Best Essay on the Theory of Scientific Change.

\section{Keywords}

theoretical scientonomy, method, methodology, theory, normative theory, descriptive theory, the third law, the first law, scientific mosaic

\section{Abstract}

The scope of the Theory of Scientific Change (TSC) encompasses any and all changes that occur in a given scientific mosaic, the set of all methods employed and theories accepted at a given time by a given scientific community. Currently, theory is defined as a set of propositions that attempts to describe something. This definition excludes normative propositions from the scope of the TSC. Normative theories, such as those of methodology or ethics, have been excluded since including them appears to give rise to a destructive paradox first identified by Joel Burkholder. There are many historical cases where employed scientific methods are known to conflict with professed methodologies. This seems to violate the third and zeroth laws of scientific change. By the third law, employed methods are deducible from accepted theories. But, this seems impossible in cases where methodologies and methods conflict. Under the zeroth law, all elements in the scientific mosaic are compatible with one another. But, that seems to be clearly not the case if methodologies and methods conflict with one another. In this paper, I argue that normative propositions such as methodologies can be included in the scientific mosaic as accepted theories without generating a paradox and that neither the third nor zeroth laws of scientific change need be violated. I outline my solution to the paradox of normative theories and conclude by describing some new and exciting avenues for future research that are now open. 
TSC: theory of scientific change

\section{Introduction}

The status of normative propositions, such as scientific methodologies, within the theory of scientific change (TSC) (Barseghyan, 2015) was called into question by the paradox of normative theories identified by Joel Burkholder (Burkholder, 2014). Here, I will argue for a solution to this paradox and show that normative propositions can be part of the scientific mosaic without having destructive consequences for the TSC. I will begin by explaining how the question of the status of methodologies in the TSC led to the identification of a paradox. I will then explain the paradox and provide a solution, thereby demonstrating how normative propositions can be given the status of theories in the TSC. I will conclude by suggesting some further avenues of research that are open now that normative propositions can be included in the TSC as theories.

A scientific mosaic is the set of all methods employed and theories accepted at a given time by a given scientific community (Barseghyan, 2015, pp. 3-5). It is constantly undergoing change as new elements are added and old ones are removed. The TSC makes a key distinction between the implicit criteria actually employed in assessing theories for inclusion in the mosaic, which are referred to as "methods", and the rules of theory assessment explicitly professed by scientists, which are referred to as "methodologies" (Barseghyan, 2015, p. 51). Although there are clear cases in the history of science where the explicit methodologies professed by scientists were quite different from the methods they actually employed in theory appraisal, this critical implicit-explicit distinction has previously often been overlooked (Barseghyan, 2015, p. 52).

\begin{tabular}{|c|}
\hline Method $\equiv$ \\
\hline $\begin{array}{c}\text { A set of implicit rules to be } \\
\text { employed in theory assessment. }\end{array}$ \\
\hline
\end{tabular}

\begin{tabular}{|c|}
\hline Methodology $\equiv$ \\
\hline $\begin{array}{c}\text { A set of explicitly formulated } \\
\text { rules of theory assessment. }\end{array}$ \\
\hline
\end{tabular}

Methodologies are normative rather than descriptive, that is, they describe the ways in which theory assessment ought to be done. The discipline of methodology is defined as a "normative discipline that formulates the rules which ought to be employed in theory assessment" (Barseghyan, 2015, p. 13). This goal is different from that of the TSC, which seeks a descriptive account of how scientific changes actually do occur (Barseghyan, 2015, pp. 12-21). But methodologies and other normative propositions are relevant to the process of scientific change and so the problem of how and whether to include them in the TSC's descriptive account is a critical one. Should they be treated as theories or as something else? Though it was initially tempting to treat normative propositions as theories, this was found to lead to a paradox, and the status of normative propositions has been problematic ever since. Currently, theory is defined as a set of propositions that attempts to "describe something", but normative propositions are prescriptions, not descriptions (Barseghyan, 2015, ch. 4). If normative propositions were to be considered theories, then the definition of theory would have to be changed so that theories could be either descriptive or normative (including those of methodology and ethics):

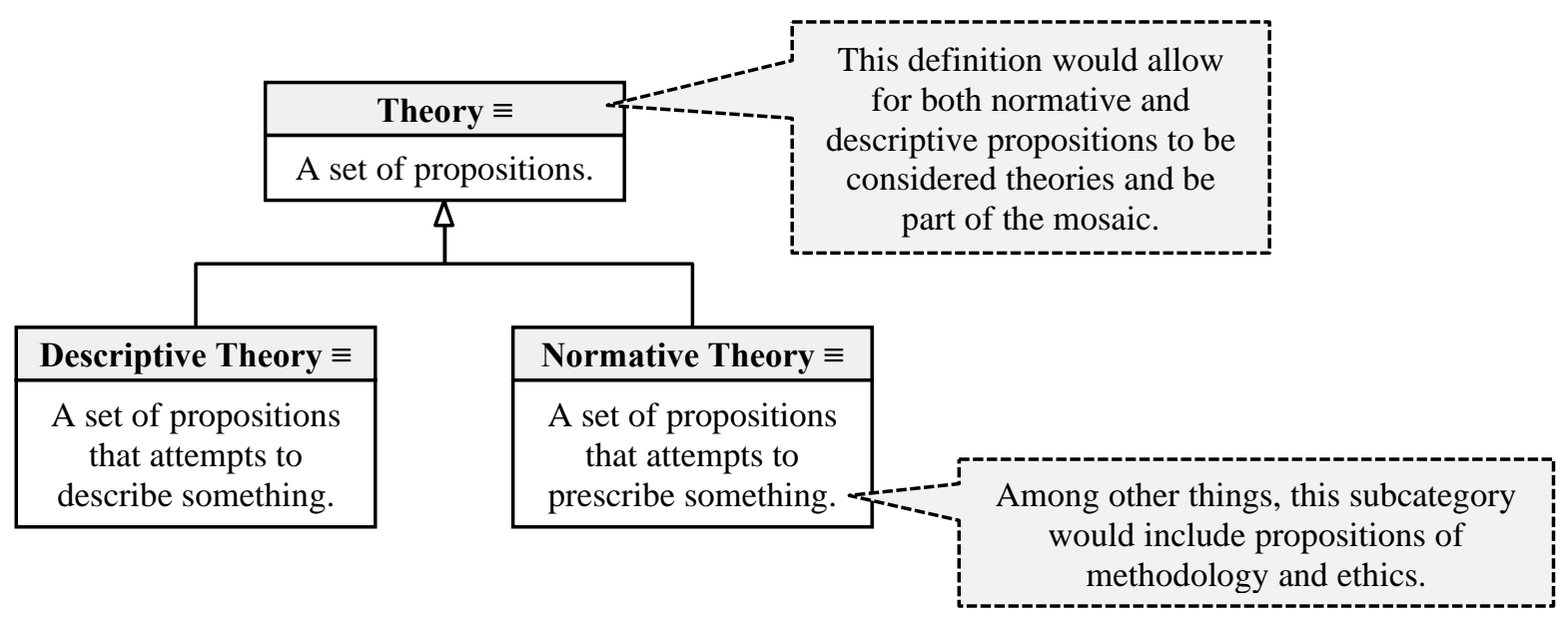


The laws of scientific change would need to be appropriately modified. If such changes are possible, the TSC would be able to include normative propositions within its scope, and an entire new area of future research on normative theories would open.

\section{The Paradox}

Unfortunately, the paradox seems to show that this way of incorporating methodologies and normative propositions into the TSC will not work. Suppose that methodologies are given the status of theories and therefore become part of the scientific mosaic. By the third law; the law of method employment, a method becomes employed "only when it is deducible from other employed methods and accepted theories of the time" (Barseghyan, 2015, p. 128).

\section{$3^{\text {rd }}$ Law: Method Employment}

A method becomes employed only when it is deducible from other employed methods and accepted theories of the time.

Since employed methods are always deductive consequences of accepted theories and since accepted methodologies are (in this picture) accepted theories, it seemingly follows that employed methods must also be deductive consequences of accepted methodologies. However, as mentioned earlier, employed methods can be very different from accepted methodologies. For example, scientists in the late $18^{\text {th }}$ and early $19^{\text {th }}$ centuries openly professed a version of the "empiricist inductivist methodology", which required that new theories be deducible from phenomena and not postulate any unobservable ("occult") entities or qualities (Nola and Sankey, 2000, p. 5; Barseghyan, 2015, p. 52). But, these same scientists accepted several theories that did postulate unobservable entities, including Franklin's theory of electricity, which postulated an unobservable electrical fluid; phlogiston chemical theory, which posited an unobservable, odourless, tasteless, massless, and colourless entity called "phlogiston" to explain combustion; and Newton's own physical theory, which postulated unobservables like gravitational attraction, absolute space, and absolute time (Barseghyan, 2015, pp. 52-53). These theories were also not deducible from phenomena and so met neither of the acceptance criteria professed (Barseghyan, 2015, p. 53). Rather, theories like that of Newton were only accepted after some of their novel predictions had been confirmed (Barseghyan, 2015, p. 48). This is a good indication that some version of the hypothetico-deductive method, which requires theories positing unobservable entities to have confirmed novel predictions, was actually employed (Irzık and Nola, 2006, pp. 231-236; Barseghyan, 2015, p. 48).

In this case, therefore, the actual expectations or method of the scientific community differed from the community's explicitly stated methodology (Barseghyan, 2015, p. 53). This is where the paradox arises, and it poses two problems. First, since the method is not a deductive consequence of the methodology, the third law of scientific change is seemingly violated. Secondly, the zeroth law, which states that the parts of the scientific mosaic must be compatible with one another, is also seemingly violated.

\section{$0^{\text {th }}$ Law: Compatibility}

At any moment of time, the elements of the scientific mosaic are compatible with each other.

A schematic version of the paradox of normative theories and the two issues it presents can be seen below: 


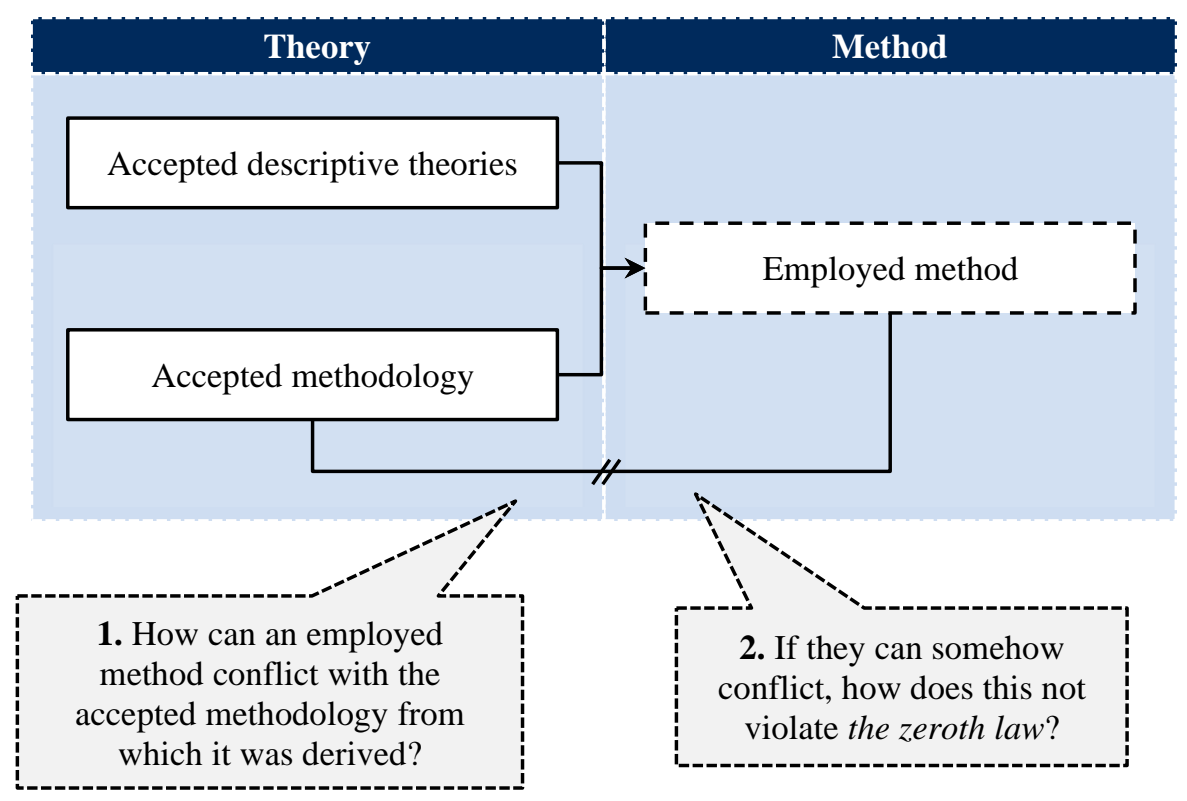

\section{Resolving the Paradox}

Due to the paradox, methodologies and all other normative propositions were left out of the scientific mosaic in Barseghyan's initial presentation of the TSC (Barseghyan, 2015, p. 60). They were not included in the definition of theories, they could not be spoken of as being accepted or unaccepted, and their status remained unknown. I will now outline my solution to the paradox and show how methodologies can be given the status of theories and included in the mosaic. To the first problem presented by the paradox, I will respond by saying that while it is true that when employed methods are the deductive consequences of accepted methodologies, those methods and methodologies cannot conflict, but it is not always the case that employed methods are the deductive consequences of accepted methodologies. By the third law, the law of method employment, a method becomes employed only when it is deducible from some other employed methods and accepted theories of the time. A method does not have to be deducible from all other employed methods and accepted theories of the time. This would be impossible, since the scientific mosaic can include millions of accepted theories, and no one person could possibly know them all. Human beings are not omniscient.

Thus, while it is true that employed methods are always deductive consequences of some accepted theories and employed methods, those from which an employed method is deducible may make up only a small portion of all the elements that are a part of the scientific mosaic at that time. There is, therefore, no paradox when a methodology that is logically inconsistent with an employed method is included in the scientific mosaic as an accepted theory. This is because, in cases like that of the Newtonian scientists, it is obvious that the accepted methodology could not have been among the accepted theories from which the employed method was deduced. Since the two were inconsistent, deducing the employed method from the accepted methodology was not possible. For purposes of clarity, I recommend refining the third law to make it clear that employed methods do not have to be deducible from all accepted theories and employed methods but only from some subset of them:

\section{$3^{\text {rd }}$ Law: Method Employment}

A method becomes employed only when it is deducible from some subset of other employed methods and accepted theories of the time.

The fact that human beings are not omniscient leads me to my next point; my response to the second problem posed by the paradox - the issue of compatibility and the possibility of violating the zeroth law. In its initial 
formulation, the zeroth law was the law of consistency, which stated that all elements of the scientific mosaic had to be logically consistent with one other. Thus, a conflict between an accepted methodology and an employed method would be a violation of the zeroth law (Barseghyan, 2015, p. 149). This formulation has been abandoned as untenable and the zeroth law has been reformulated as the law of compatibility (Harder, 2013; Barseghyan, 2015, p. 149). Limited human minds cannot possibly ensure that all elements of the scientific mosaic are logically consistent with one another. In complex scientific mosaics it is well-nigh impossible to ensure that all the openly accepted propositions are mutually consistent, and even if this were somehow possible, there would remain the possibility that the logical consequences of any two accepted theories are mutually inconsistent (Barseghyan, 2015, p. 149).

Besides the limitations of the human mind, there are many examples where the scientific community knowingly allows two logically inconsistent elements to be a part of the same mosaic at the same time, because their inconsistency does not render them incompatible. General relativity and quantum physics are inconsistent with one another, but have both been accepted because they deal with largely distinct domains of phenomena, and are therefore deemed compatible (Barseghyan, 2015, pp. 11, 150). Bueno and da Costa (2007) have noted that inconsistencies often arise since we have incomplete knowledge. They do not pose a problem for science since we ascribe the status of "quasi-truth" to our theories to indicate that they do not describe an entire domain, but only one aspect of it - an aspect that we can model with a "partial structure" (Bueno and da Costa, 2007, p. 388). By using partial relations and partial structures to represent our "quasi-true" knowledge, elements in our scientific mosaic that conflict can still be compatible with one another since they are only "quasi-true" and not absolutely true (Bueno and da Costa, 2007, p. 392).

Brewer and Chinn (1994) also discuss the fact that scientific communities sometimes knowingly accept anomalous data that conflicts with an accepted theory without rejecting either the theory or the data (Brewer and Chinn, 1994, p. 305). In such cases, they explain, the scientific community excludes the data from the theory's domain, thereby allowing the two to remain in the scientific mosaic and be compatible with one another even though they are not logically consistent (Brewer and Chinn, 1994, p. 307). Thagard (1992) argues that this was the case with geophysics and geology around the middle of the twentieth century (Brewer and Chinn, 1994, p. 307). While twentieth century geophysicists accepted the principle of isostasy (that the earth's crust is in "gravitational equilibrium" and has not been recently disturbed), geologists posited that land bridges rose and fell periodically between the continents (which would explain why fossils on opposite sides of oceans are similar) (Brewer and Chinn, 1994, p. 307). Thagard (1992) argues that in this case the conflict between these theories was tolerated since "they belonged to different disciplines" and Kuhn (1962) likewise noted that scientists sometimes respond to anomalies by treating them as the "concern of another discipline" (Brewer and Chinn, 1994, p. 307).

These philosophical and historical arguments are the motivation behind the zeroth law being formulated as the law of compatibility, which now states that all elements of the scientific mosaic must be compatible rather than logically consistent with one another (Barseghyan, 2015, p. 149). Since compatibility does not entail consistency, conflicts between accepted methodologies and employed methods do not violate the current formulation of the zeroth law and the paradox of normative theories dissolves. Methodologies can, therefore, be included in the definition of theories and seen as part of the scientific mosaic without difficulty. Moreover, for historical cases like that of the Newtonian scientists, there is no paradox generated by the fact that their accepted methodology (an accepted theory) conflicted with their employed method (another element of their scientific mosaic). The question of how these scientists justified the compatibility of their methods with their methodology is, however, an important matter for historical research. I summarize my solution to the paradox of normative theories schematically: 


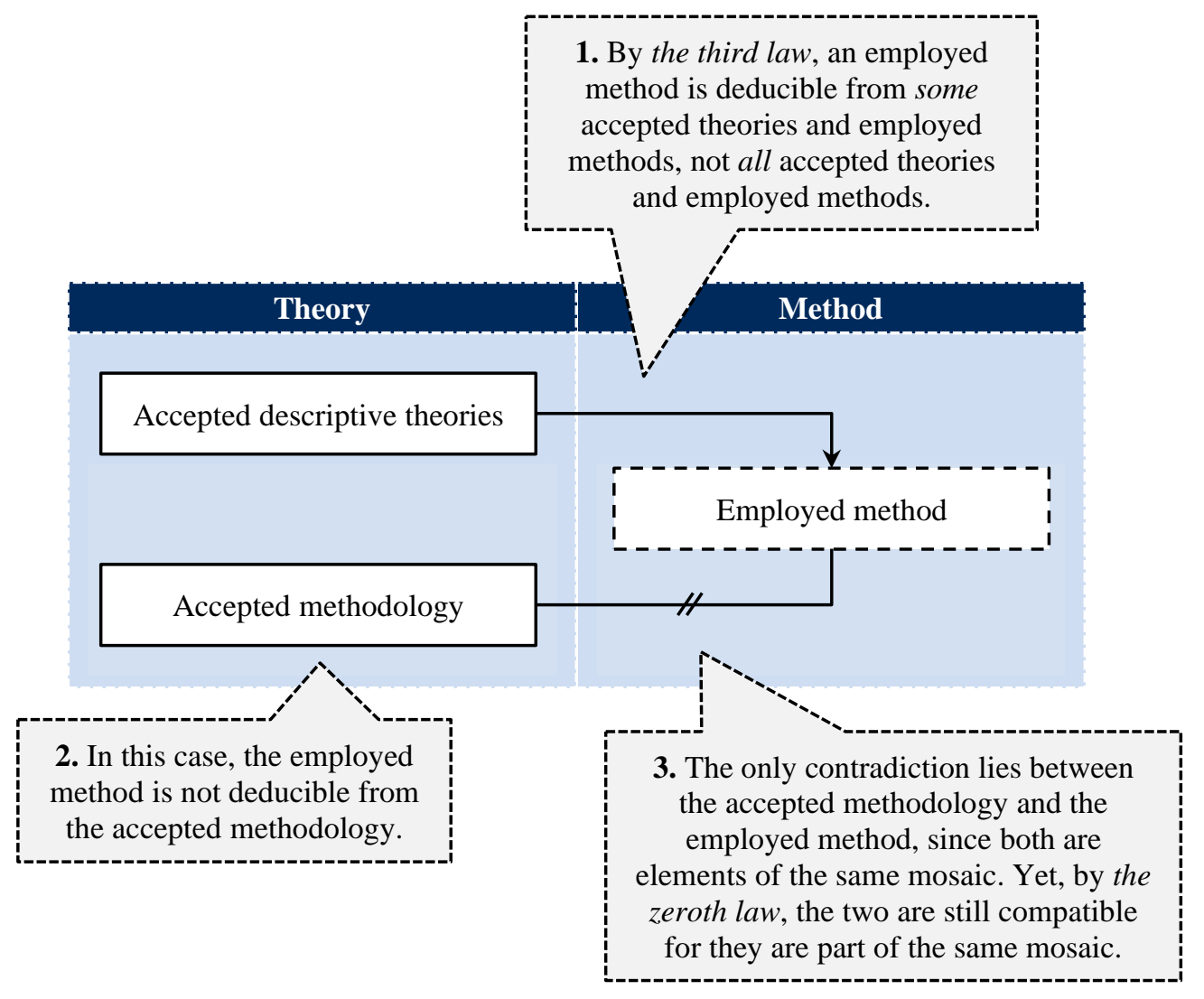

\section{Consequences of the Resolution}

Based on my solution to the paradox of normative theories, methodologies, as well as all other normative propositions, can be seen as theories within the scientific mosaic. Their assessment, acceptance, and rejection can now be understood using the same framework as for other theories in the TSC. To do this, the TSC's definition of theory must be changed to include normative as well as descriptive propositions. I recommend defining theory as "a set of propositions" so that both normative and descriptive propositions are included in the definition. Consequently, there will be two subcategories of theories, descriptive and normative, and methodology will be a subcategory of normative theory:

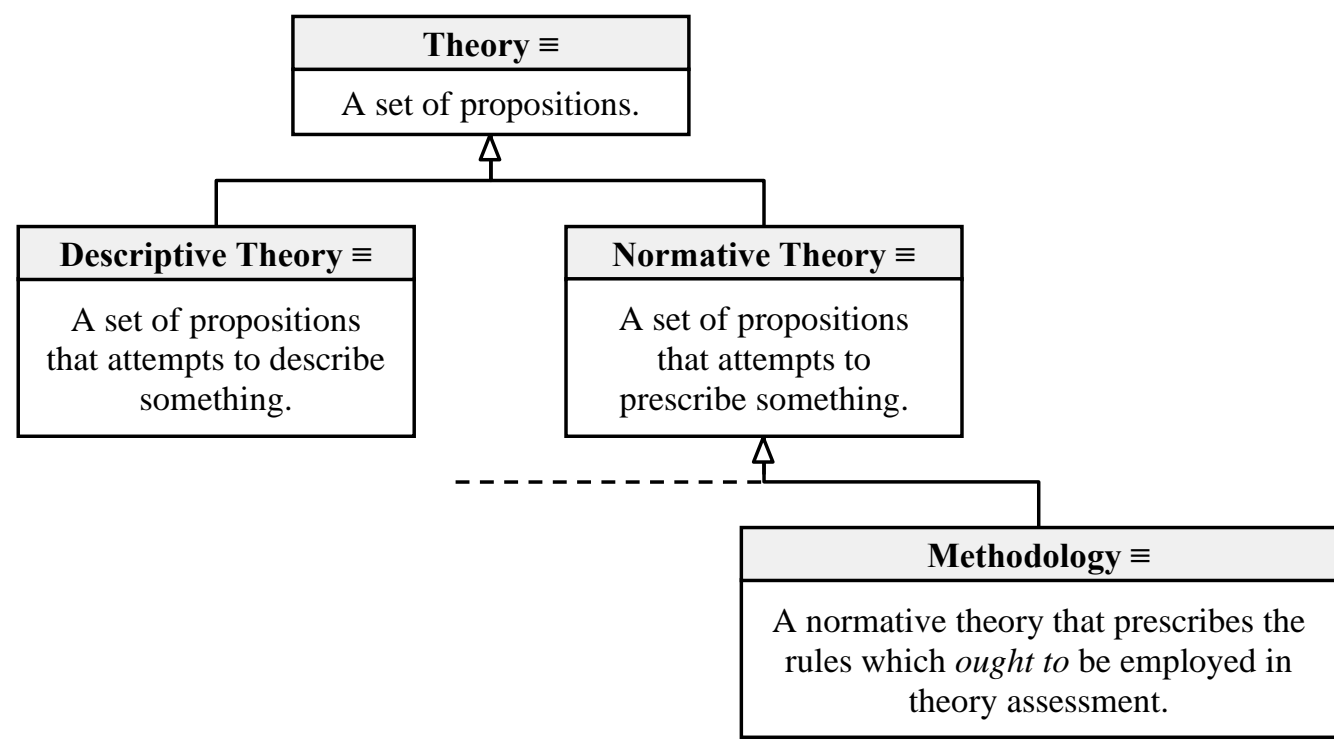


By allowing us to add normative propositions to the TSC, the solution to the paradox opens a vast new field of inquiry. One interesting avenue of study is the nature of the influence that methodologies exert over employed methods. Now that accepted methodologies are a part of the mosaic, the TSC can study how accepted methodologies affect which methods come to be employed. It can also determine whether employed methods were derived from accepted methodologies, and how the employed methods are rendered compatible with accepted methodologies even when the two logically conflict with one another. The TSC can now also study how methodologies come to be pursued, accepted, and rejected. Methodologies, like all other theories, can be accepted only once they satisfy the requirements of whatever method is employed at the time, and can be rejected only when other theories incompatible with them become accepted (Barseghyan, 2015, p. 168). This assumes that the definition of theory acceptance should be modified to make it possible for normative propositions to be accepted:

\begin{tabular}{|c|}
\hline Theory Acceptance $\equiv$ \\
\hline $\begin{array}{c}\text { A theory is said to be accepted if it is } \\
\text { taken as the best available description } \\
\text { or prescription of its object. }\end{array}$ \\
\hline
\end{tabular}

Now that normative propositions can be understood within the framework of the TSC, ethical propositions can be brought within its compass. The TSC can study how ethical propositions come to be accepted into the scientific mosaic and the effects they have on which methods come to be employed. Indeed, including normative theories like ethics into the TSC may help to develop theorems regarding which concrete method (of those available) becomes employed to satisfy the requirements of whatever abstract method followed directly from the accepted theories. As it stands now, the TSC recognizes that the same abstract requirement can be implemented in different ways, but there seems to be "a certain creative gap between abstract requirements that follow directly from accepted theories and methods that implement these abstract requirements" (Barseghyan, 2015, p. 147).

The blind trial method, for example, is one way of implementing the abstract requirement that the placebo effect must be taken into account when assessing a drug's efficacy (Barseghyan, 2015, p. 135). There are alternatives, however, that can be used instead. One such alternative is to conceal the treatment in the patient's food or drink, so that they are unaware they are taking the drug. Another, if we had nanotechnology capable of tracing all the molecules of a drug in a patient's body, would be to use nanotechnology to ensure that any improvement in the patient's condition has to do with the drug and not the placebo effect (Barseghyan, 2015, p. 135). While the last option cannot be used because the supposed technology does not yet exist, accepted ethical theories about whether or not it is morally appropriate to slip drugs in someone's food or drink without them knowing may account for why the second option is not used in practice. Indeed, the second alternative would likely be much more efficient than the blind trial method (tricking people into taking drugs would likely get more people testing the drug more easily and more cheaply than setting up a blind trial would) and yet we opt for the blind trial method instead. It would be interesting to see what kind of insights could be gleaned from studying normative propositions and the ways in which they might affect which concrete methods are chosen for use in actual practice.

\section{Conclusion}

My solution to the paradox of normative theories allows normative propositions, including methodologies, to be included in the scientific mosaic as accepted theories without having any destructive consequences for the TSC. This is because in a situation where an accepted methodology is logically inconsistent with an employed method, I have shown that no paradox arises for either the third law or the zeroth law. The third law stipulates only that employed methods must be deducible from some subset, not all, of the accepted theories in the mosaic. So, in such a case, it must be that the employed method was not deduced from the methodology it conflicts with but from some other accepted theories - theories with which the method does not conflict. Moreover, there is no violation of the zeroth law since, in its current formulation, the zeroth law requires only that all the elements in 
the mosaic be compatible with one another, and two elements can be inconsistent while still being mutually compatible. The paradox is thus solved and all normative propositions, including methodologies, can be given the status of "theories" under the TSC. Ascribing the status of "theories" to normative propositions and methodologies will greatly expand the scope of the TSC. It opens entirely new avenues for research and suggests that it may have interdisciplinary value extending far beyond its origin in studies of the history and philosophy of science.

\section{Suggested Modifications}

Thus, I suggest the following modifications:

\section{[Sciento-2016-0001]}

Accept the following reformulation of the third law:

- The Third Law: a method becomes employed only when it is deducible from some subset of other employed methods and accepted theories of the time.

\section{$3^{\text {rd }}$ Law: Method Employment}

A method becomes employed only when it is deducible from some subset of other employed methods and accepted theories of the time.

Consequently, accept that there is no paradox of normative theories: when an employed method and an accepted methodology are logically inconsistent with one another, it merely indicates that the employed method isn't a logical consequence of the accepted methodology. By the third law, the employed method still follows from some accepted theories, but not from this particular methodology.

Reject the previous formulation of the third law; it can remain in use for educational purposes.

\section{[Sciento-2016-0002]}

Provided that the preceding modification [Sciento-2016-0001] is accepted, accept the following taxonomy for theory, descriptive theory, normative theory, and methodology:

- $\quad$ Theory $\equiv$ a set of propositions.

- Descriptive Theory $\equiv$ a theory that attempts to describe something.

- Normative Theory $\equiv$ a theory that attempts to prescribe something.

- Methodology $\equiv$ a normative theory that prescribes the rules which ought to be employed in theory assessment.

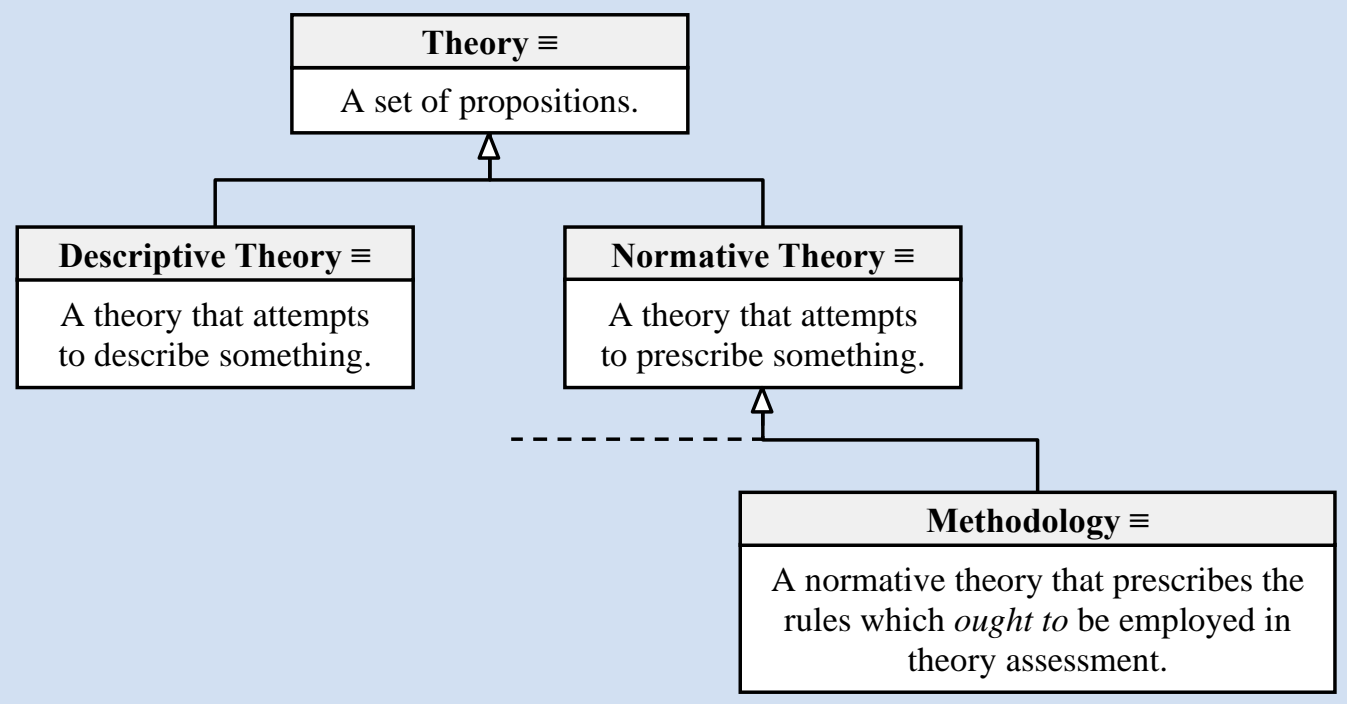


Modify the definition of theory acceptance to make it possible for both descriptive and normative theories to be accepted:

- Theory Acceptance $\equiv$ a theory is said to be accepted if it is taken as the best available description or prescription of its object.

\section{Theory Acceptance $\equiv$}

A theory is said to be accepted if it is taken as the best available description or prescription of its object.

Reject the previous definitions of theory, methodology, and theory acceptance.

\section{References}

Barseghyan, H. (2015). The Laws of Scientific Change. Springer.

Brewer, W. F. \& Chinn, C. A. (1994). Scientists' Responses to Anomalous Data: Evidence from Psychology, History, and Philosophy of Science. PSA: Proceedings of the Biennial Meeting of the Philosophy of Science Association, Vol. 1994, Volume One: Contributed Papers. University of Chicago Press, pp. 304-313.

Bueno, O. \& da Costa, N. C. A. (2007). Quasi-truth, Paraconsistency, and the Foundations of Science. Synthese 154, pp. 383399.

Burkholder, J. (2014). Protomethod, the Third Law, and Ethical Propositions. Unpublished.

Harder, R. (2013). Scientific Mosaics and the Law of Consistency. Unpublished.

Irzık, G. \& Nola, R. (2006). Philosophy, Science, Education \& Culture. Springer.

Kuhn, T. (1962). The Structure of Scientific Revolutions. The University of Chicago Press, 1996.

Nola, R. \& Sankey, H. (2000). A Selective Survey of Theories of Scientific Method. In Nola \& Sankey (Eds.) (2000), pp. 165.

Nola, R. \& Sankey, H. (Eds.) (2000). After Popper, Kuhn, and Feyerabend. Recent Issues in Theories of Scientific Method. Kluwer Academic Publishers.

Thagard, P. (1992). Conceptual Revolutions. Princeton University Press. 